\title{
COOPERATIVE ADAPTIVE DRIVING FOR PLATOONING AUTONOMOUS SELF DRIVING BASED ON EDGE COMPUTING
}

\author{
BEN-JYE CHANG ${ }^{a}$, REN-HUNG HWANG ${ }^{b, *}$, YUEH-Lin TSAI $^{a}$, BO-HAN YU $^{a}$, \\ YING-HSIN LIANG ${ }^{c}$
}

\author{
${ }^{a}$ Department of Computer Science and Information Engineering \\ National Yunlin University of Science and Technology \\ 123 University Rd., Sec. 3, Douliou, Yunlin 64002, Taiwan, Republic of China \\ ${ }^{b}$ Department of Computer Science and Information Engineering \\ National Chung Cheng University \\ 168, University Rd., Sec. 1, Minhsiung, Chiayi 62102, Taiwan, Republic of China \\ e-mail: rhhwangecs.ccu.edu.tw \\ ${ }^{c}$ Department of Multimedia Animation and Application \\ Nan Kai University of Technology \\ 568, Zhongzheng Rd., Caotun, Nantou, 542, Taiwan, Republic of China
}

\begin{abstract}
Cooperative adaptive cruise control (CACC) for human and autonomous self-driving aims to achieve active safe driving that avoids vehicle accidents or traffic jam by exchanging the road traffic information (e.g., traffic flow, traffic density, velocity variation, etc.) among neighbor vehicles. However, in CACC, the butterfly effect is encountered while exhibiting asynchronous brakes that easily lead to backward shock-waves and are difficult to remove. Several critical issues should be addressed in CACC, including (i) difficulties with adaptive steering of the inter-vehicle distances among neighbor vehicles and the vehicle speed, (ii) the butterfly effect, (iii) unstable vehicle traffic flow, etc. To address the above issues in CACC, this paper proposes the mobile edge computing-based vehicular cloud of the cooperative adaptive driving (CAD) approach to avoid shock-waves efficiently in platoon driving. Numerical results demonstrate that the CAD approach outperforms the compared techniques in the number of shock-waves, average vehicle velocity, average travel time and time to collision (TTC). Additionally, the adaptive platoon length is determined according to the traffic information gathered from the global and local clouds.
\end{abstract}

Keywords: mobile edge computing, active safe driving, cooperative platoon driving, cooperative adaptive cruise control.

\section{Introduction}

This section first briefly describes cooperative active safe driving (Burke and Williams, 2012) for achieving active safe driving, and then depicts adaptive cruise control (ACC) and cooperative adaptive cruise control (CACC). Furthermore, we classify the critical issues into two types, and the related studies are listed below.

1.1. Active safe driving. The work of Burke and Williams (2012), extended from ITS (2018), adopts VANET using wireless networking: IEEE 802.11p

${ }^{*}$ Corresponding author
(IEEE, 2011; 2013) or LTE-V (3GPP, 2019; Chen et al., 2016), as the information sharing network for gathering the information on the vehicular traffic and road situation, and then cooperative-ITS (C-ITS) enables the cooperative mechanism for achieving active safe driving. Several driver-assist mechanisms: advanced driver-assistance systems (ADASs) (Maag et al., 2012), ACC (Pauwelussen and Feenstra, 2010; Stevenson, 2011), and CACC (Papadimitratos et al., 2009; Desjardinsand and Chaib-draa, 2011) are proposed to assist the driver by using various types of sensors (e.g., a radar, image scanner, video detector, etc.) to alarm drivers or to directly involve assistant driving control while encountering 
explicit and implicit dangers. However, they suffer from sudden braking and unstable driving. The asynchronous braking and the stop-and-go results of the preceding vehicles easily yield a significant impact on the driving speed of the succeeding vehicles. The phenomenon is called the butterfly effect (Lorenz, 1963) and transfers a backward shockwave on the behind vehicle flow and may cause danger. In distributed assistant driving control (e.g., CACC), several critical issues should be addressed to achieve safely active safe driving, including (i) a stable safe time headway, (ii) a stable car-following distance or a stable inter-vehicle distance, (iii) the minimum and maximum driving speeds of different-class of roads, etc. The related works on assistant driving control can be classified into two types: (i) cooperative warning and maneuvering (Mitropoulos et al., 2010; Huang et al., 2010; Taleb et al., 2010; Milanés et al., 2011a; 2011b), and (ii) traffic flow stability and shockwave analysis (Asadi and Vahidi, 2011; Geroliminis and Skabardonis, 2011; Schakel et al., 2010; Wilmink et al., 2007; Somda and Cormerais, 2011). Furthermore, recently, the mobile edge computing (MEC) mechanism (Gomes et al., 2017; Dinh et al., 2017; Satria et al., 2017) has been specified to minimize the transmission delay by moving the computing-intensive and data-intensive virtual machine instances of the global cloud to the local site that is near the users. Extensively, the MEC mechanism can be combined and cooperated with the vehicular cloud.

1.2. Critical issues, motivations, and objectives. From related works on cooperative driving control, several critical issues occur in CACC for active safe driving. The problems below need be addressed effectively before achieving an efficient cooperative control for active safe driving.

1. Regarding CACC, several works neglect the transmission delay of each wireless link on the packet routing path, affecting the accuracy of shockwave analysis (Mitropoulos et al., 2010; Wilmink et al., 2007).

2. The butterfly effect yielding backward shockwaves and an unstable vehicle traffic flow are difficult to be addressed in a fully distributed VANET (Milanés et al., 2011b; Geroliminis and Skabardonis, 2011; Wilmink et al., 2007).

3. Most works only concern the average vehicle velocity, rather than some key parameters: traffic flow, traffic density, velocity variation (Taleb et al., 2010; Milanés et al., 2011a; Schakel et al., 2010; Somda and Cormerais, 2011).

This paper aims to propose a platoon-based cooperative approach, extended from the work of Hwang et al. (2017), which consists of three phases, to analyze and detect driving backward shockwaves, to avoid unstable driving, and to increase driving safety and time-to-collision (TTC). The significant contributions include:

1. minimizing the backward shockwaves and the butterfly effects problem in active safe driving,

2. adopting an adaptive platoon synchronization domain to dynamically determine the velocity and the platoon length,

3. increasing the driving stability and minimizing the waste of gasoline,

4. achieving active safety driving efficiently,

5. increasing road utilization, etc.

Note that in platoon-based CACC the platoon length is defined as the maximum length of a vehicle group, i.e., the distance from the first vehicle member to the last one.

The reminder of this paper is organized as follows. The network model is defined in Section 2. Section 3 details the proposed cooperative adaptive driving (CAD) approach. Numerical results of simulations are provided in Section 4. Conclusions are given in Section 5.

\section{Network model}

A vehicular network is defined as a directed graph $G=$ $(V, E)$ that consists of a set of mobile vehicles $V$ and a set of wireless links $E$, as demonstrated in Fig. 1. Some vehicles equipped with the cooperative adaptive driving (CAD) system are denoted as $V_{j}^{\text {CAD }}$ (or $V_{j}$ ), where $j$ represents the vehicle index, i.e., the light grey vehicles that install the CAD system and a wireless network and offer various types of sensors to sense vehicle traffic or environmental objects (including all types of vehicles). Conversely, some vehicles not equipped with CAD are denoted as $V_{k}^{\text {Non-CAD }}$, as shown as the dark grey vehicles

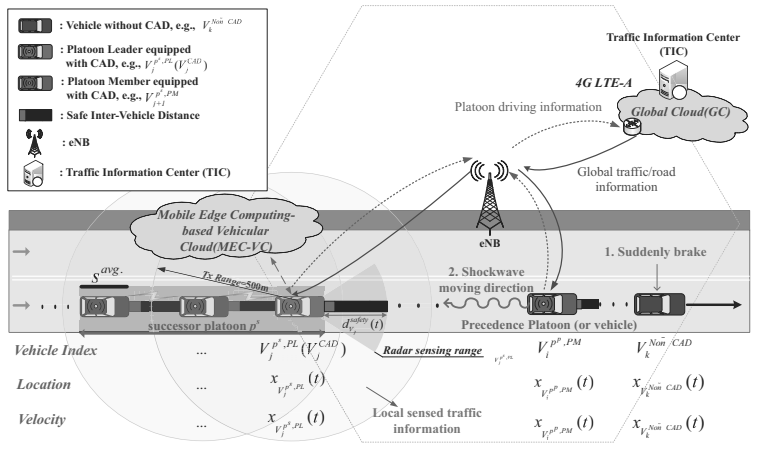

Fig. 1. Network model. 
that can be sensed by $V^{\mathrm{CAD}}$ but cannot communicate with any vehicles because of the lack of the wireless interface.

By using wireless communications, two types of clouds are formed: the global cloud (GC) and the vehicular cloud (VC) (Abuelela and Olariu, 2010; Mousannif et al., 2011). CAD vehicles can receive global traffic information from the GC and the real-time-based local traffic information from the VC managed by the platoon. The managed traffic information consists of spatial and time localities-based information, such as the platoon identifier (PID), vehicle ID, geographical location, velocity, the limited speed of roads, etc.

In the vehicle platoon model, for any two adjacent platoons, $p^{s}$ denotes the succeeding platoon and $p^{p}$ the preceding platoon. The average velocity and the length of the platoon (e.g., $p^{s}$ ) at time $t$ are denoted as $\bar{v}_{p^{s}}(t)$ and $L_{p^{s}}(t)$, respectively. $N_{p^{s}}(t)$ denotes the total number vehicles of platoon $p^{s}$ at time $t$.

A platoon $p$, consists of two types of vehicles: the platoon leader (e.g., $V_{j}^{p^{s}}$, $\mathrm{PL}$ ) and the platoon members. Thus, the geographical location and velocity of the platoon leader $j$ at time $t$ are denoted as $x_{V_{j}^{p^{s} \text {, PL }}}(t)$ and $\dot{x}_{V_{j}^{p^{s}, \mathrm{PL}}}(t)$, respectively. Moreover, the distance to the ahead adjacent vehicle is sensed by the radar sensor and denoted as $\Phi_{V_{j}^{p^{s}, P L}}$. To guarantee safe driving, $\Phi_{V_{j}^{p^{s} \text {, PL }}}$ should be smaller than the determined safe inter-vehicle distance at time $t, d_{V_{j}}^{\mathrm{Safe}}(t) . \quad d_{v_{j}}^{\mathrm{Safe}}(t)$ is dynamically determined according to three parameters: (i) the moving distance after a response at time $t$, denoted as $d_{V_{j}}^{\text {Response }}(t)$, (ii) the required distance of a full stop at time $t$, denoted as $d_{V_{j}}^{\text {Brake }}(t)$, and (iii) the distance for the transmission of synchronization message at time $t$, denoted as $d_{V_{j}}^{\text {Sync }}(t)$. Furthermore, we assume that the minimum distance of the standstill vehicle is $s^{\text {bumper }}$.

Additionally, when the traffic flow $\left(d_{p^{p}}\right)$ and the average vehicle velocity $\left(\bar{v}_{p^{p}}\right)$ of the preceding platoon decrease and the vehicle density $\left(k_{p^{p}}\right)$ increases, the successor platoon, $p^{s}$, easily yields a backward shockwave. The shockwave speed, $W_{p^{p-s}}^{V}$, can be determined by the differences of the traffic flow, the average vehicle velocity, and the vehicle density of the successor and the preceding platoons.

\section{Proposed CAD approach}

This section details the proposed approach of cooperative adaptive driving (CAD) that consists of three phases.

\subsection{Phase 1: Cooperative vehicle platooning (CVP).} For minimizing the butterfly effect and shockwaves, CAD proposes an adaptive management for cooperative vehicle platooning (CVP) driving as the key element for realizing CAD, extended from the work of Chang et al. (2017). Figure 2 demonstrates that the vehicles of a platoon $p^{s}$

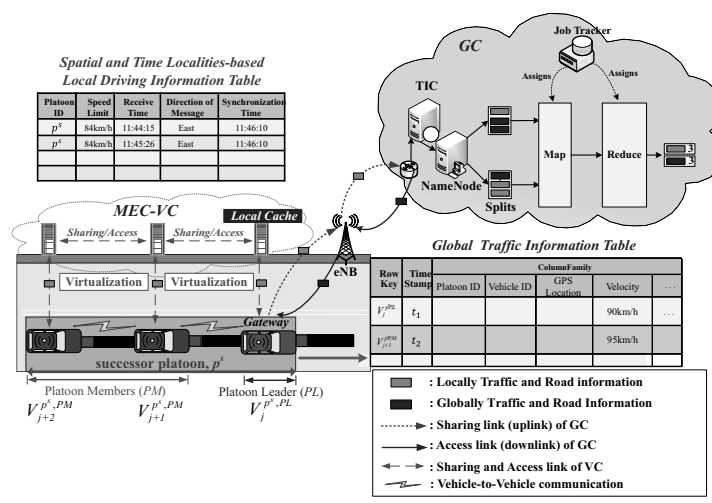

Fig. 2. Driving state of CAD.

exhibit two roles: (i) a platoon leader (e.g., $V_{j}^{p^{s}, \mathrm{PL}}$ ) and (ii) platoon members (e.g., $V_{j+1}^{p^{s}, \mathrm{PM}}$ ).

3.1.1. Platoon leader (PL) determination. In CAD, for minimizing the computing and transmission delay, the mobile edge computing (MEC) mechanism (Gomes et al., 2017; Dinh et al., 2017; Satria et al., 2017) is adopted to move the computing-intensive and data-intensive virtual machine instances of the global cloud to the local site that is closer to vehicles. Furthermore, the MEC mechanism is combined and cooperates with the vehicular cloud (namely VC or MEC-VC) to achieve real-time computing, to minimize transmission delay, and to allocate/re-direct the optimal VM instances of diverse applications to the platoon.

Thus, in $\mathrm{CAD}$, the first vehicle forming a platoon or the vehicle with the least MAC address will be selected as the platoon leader (PL) when the platoon is initialized. The PL manages the entire platoon synchronization and maintains the platoon traffic information of each platoon member in a local vehicle cloud (VC) table, as shown in Fig. 2 and detailed below.

First, the PL manages the platoon members when some of them join/leave the platoon. Second, by using a local vehicle cloud, PL maintains the sensed/received traffic information gathered from the shared broadcast messages sent by PMs. Note that the sharing messages with a timestamp are forwarded within a limited hop count, i.e., the synchronization platoon length $L_{p^{s}}(t)$, determined by Phase 3. Third, the PL determines the platoon velocity and synchronous length, and then broadcasts the up-to-date control message to all PMs periodically or on demand. Thus, the PMs can synchronously adjust their velocity at the next synchronization time $\tau_{V_{j}^{p^{s, \mathrm{PL}}} \text {. }}^{\text {Sync }}$.

For timely keeping and updating the local real-time traffic information in the local MEC-VC, the cooperation 
as a service (CaaS) cloud computing mechanism is proposed herein and managed by the PL, as demonstrated in Fig. 2. The local VC keeps the dynamic driving state of each PM, including (i) platoon ID, (ii) vehicle ID, (iii) GPS location, (iv) vehicle velocity, (v) the highest velocity in history, (vi) limited speed, (vii) physical inter-distance, (viii) logical inter-distance, (ix) timestamp, (x) message ID, etc. Additionally, a global cloud (GC) is adopted for all vehicles of $V^{\mathrm{CAD}}$ located on different areas/roads sharing the traffic information, and thus the GC provides the global traffic and road information for every vehicle of $V^{\mathrm{CAD}}$.

The main different functions of the $\mathrm{GC}$ and $\mathrm{VC}$ are compared below. The GC is a global-based cloud computing service that provides the global traffic/road information as completely as possible, but the global information may not be updated frequently. Conversely, the $\mathrm{VC}$ is local-based cloud computing service that provides the local traffic/road information as fast as possible, but the local information may exclude the global one. Thus, by using the hybrid cloud computing services of the MEC-VC and GC, the proposed cooperative platoon approach can offer both the completely global information and the real-time local information.

Furthermore, the PL determines the platoon velocity and synchronous length according to the traffic information. All PMs of the same platoon drive based on the received information. Note that the platoon length and velocity are determined adaptively. As a result, CAD obviously minimizes asynchronous brakes and avoids driving backward shockwaves.

3.1.2. Platoon member (PM) determination. In a platoon, a PM periodically sends beacon with its driving states to neighbors, and thus the PL can finally gather the driving information of all PMs within the platoon. The gathered driving information includes (i) vehicle ID, (ii) geographical location, (iii) current velocity, (iv) highest velocity in history, (v) physical inter-distance, and (vi) receive time (timestamp). Furthermore, the PM adjusts the velocity and synchronization time after receiving the indication message sent from the PL.

Several platoon manage mechanisms are depicted as follows. First, when the current PL leaves the platoon, the PM located in the first one will be selected as the new PL because the PL of a platoon needs to lead the entire platoon moving forward. Second, when a vehicle with CAD moves alone, i.e., with no neighbors around it, it initializes the PL selection procedure to select itself as the PL. Third, when two adjacent platoons move as closely as possible, the succeeding one will be merged into the preceding one.

The consecutive steps of Phase 1 (CVP) are demonstrated as Algorithm 1.

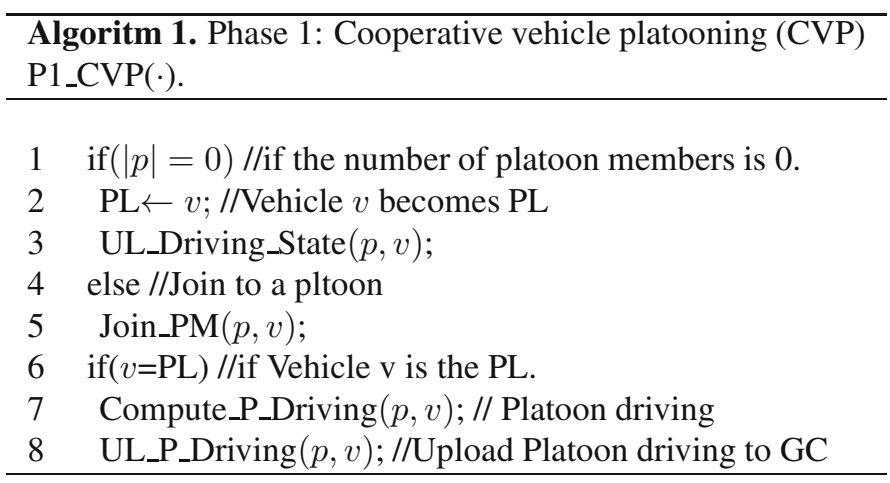

3.2. Phase 2: Shockwave-avoidance driving (SAD) In Phase 1, the platoon is initialized and managed well. For avoiding shockwaves occurring in the cooperative platoon-based driving, this section details SAD, which consists of two sub-phases: (i) maximum and minimum platoon velocities and (ii) dynamic safe car-following distance.

\subsubsection{Phase 2-1: Maximum and minimum pla-} toon velocities. In Phase 2 (SAD), the main objective is to control the platoon moving stably safely. Two important factors affect the platoon stability: (i) a dynamic platoon velocity for all PMs of the platoon and (ii) a dynamic platoon length for message synchronization. The determination of these dynamic factors for a platoon is depicted in Phase 2-1 below.

In Fig. 3, in the case of non-platoon driving, a successive vehicle is affected by the preceding vehicle. Similarly, in the case of platoon driving, a succeeding platoon $\left(p^{s}\right)$ is affected by the preceding platoon $\left(p^{p}\right)$. Clearly, the shockwaves occurring between two platoons result from the inter-platoon operation. Thus, for cooperative platoon driving, we first determine the traffic flow, traffic density and average vehicle velocity of two adjacent platoons based on traffic flow theory (Kuhne and Michalopoulos, 1997; Helbing, 2001). The traffic flow is formulated as

$$
q(t)=k(t) \bar{v}(t)
$$

where $k(t)$ is the traffic density at time $t$ and $\bar{v}(t)$ is the average velocity at time $t$. The traffic density $k(t)$ can be defined as $k(t)=N(t) / L(t)$, where $N(t)$ is the total number of vehicles on a road segment or within a platoon and $L(t)$ is the length of a road segment or the platoon length at time $t$. The speed of a shockwave can be determined by (Kuhne and Michalopoulos, 1997; Helbing, 2001)

$$
W_{p^{p-s}}^{V}(t)=\frac{q_{p^{p}}(t)-q_{p^{s}}(t)}{k_{p^{p}}(t)-k_{p^{s}}(t)}
$$

where the traffic flow of the successor and precedence platoons $\left(q_{p^{s}}(t)\right.$ and $\left.q_{p^{p}}(t)\right)$ can be obtained by Eqn. (1). 


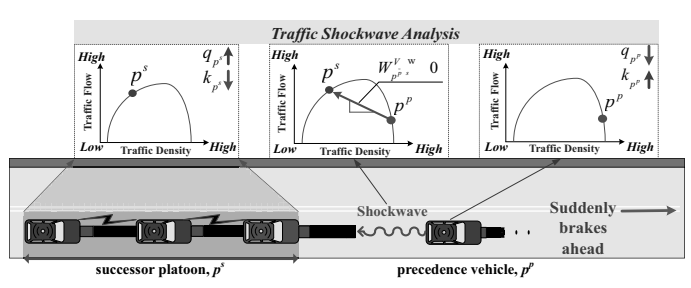

Fig. 3. Driving state of the CAD approach.

The traffic density of the succeeding and preceding platoons at time $t\left(k_{p^{s}}(t)\right.$ and $\left.k_{p^{p}}(t)\right)$ is the determined parameter. In consequence, a backward shockwave at time $t$, i.e., $W_{p^{p-s}}^{V}(t)<0$, occurs in the following conditions: (i) $q_{p^{p}}(t)<q_{p^{s}}(t)$, (ii) $k_{p^{p}}(t)>k_{p^{s}}(t)$ and (iii) $\bar{v}_{p^{p}}(t)<\bar{v}_{p^{s}}(t)$. Clearly, to avoid a shockwave, the speed of a shockwave should not be a negative value, i.e., $W_{p^{p-s}}^{V}(t) \geq 0$. We have

$$
\begin{aligned}
W_{p^{p-s}}^{V}(t) & =\frac{q_{p^{p}}(t)-q_{p^{s}}(t)}{k_{p^{p}}(t)-k_{p^{s}}(t)} \\
& =\frac{\frac{N_{p^{p}}(t) \bar{v}_{p} p(t)}{L_{p^{p}}(t)}-\frac{N_{p^{s}}(t) \bar{v}_{p^{s}}(t)}{L_{p^{s}}(t)}}{\frac{N_{p} p(t)}{L_{p} p(t)}-\frac{N_{p^{s}}(t)}{L_{p^{s}}(t)}}>1 .
\end{aligned}
$$

The maximum platoon velocity $\left(\bar{v}_{p^{s}}^{\max }(t)\right)$ at time $t$ of the succeeding platoon $\left(p^{s}\right)$ can be derived from Eqn. (3),

$$
\bar{v}_{p^{s}}^{\max }(t) \leq-\left[\frac{N_{p^{p}}(t) L_{p^{s}}(t)\left(1-\bar{v}_{p^{p}}(t)\right)}{N_{p^{s}}(t) L_{p^{p}}(t)}-1\right] .
$$

Additionally, for increasing the driving efficiently, the minimum platoon velocity needs to be determined according to the local driving information and the history information,

$$
\bar{v}_{p^{s}}^{\min }(t) \geq \min _{\forall N_{p^{s}}(t)}\{\text { the highest velocity in history\} }
$$

where $N_{p^{s}}(t)$ is the number of vehicles of the succeeding platoon $\left(p^{s}\right)$ at time $t$.

\subsubsection{Phase 2-2: Dynamic safe car-following dis-} tance. For safe driving, a dynamic safe car-following distance is an important factor to be determined for every vehicle. A high speed vehicle needs a longer safe car-following distance, and vice versa. This paper proposes a dynamic safe car-following distance for cooperative platoon driving, as detailed below.

In CAD, a platoon leader (PL) adopts the synchronization message to send the platoon synchronization time and to maximum platoon velocity to all PMs for minimizing the shockwaves and the butterfly effects. Before detailing Phase 2-2, we first introduce following notation: the shockwave affection range, $W_{p^{p-s}}^{R}(t)$, and the synchronous message distance, $d_{V_{j}^{p, \mathrm{PL}}}^{\text {Sync }}(t)$. First, the (backward) shockwave can be detected by the PL $x_{V_{j}^{p^{s}, \mathrm{PL}}}(t)$, and the shockwave affection range, $W_{p^{p-s}}^{R}(t)$, can be estimated by the shockwave speed, $W_{p^{p-s}}^{V}(t)$, as shown in Eqns. (3) and (9).

Second, the shockwave affection range, $W_{p^{p-s}}^{R}(t)$, will be adopted for the determination of the synchronous message distance, $\left(d_{V_{j}^{p, \mathrm{PL}}}^{\text {Sync }}(t)\right)$, as shown in Case 3 in this section. Note that the synchronization length should be shorter than the shockwave affection range, i.e., $d_{V_{j}^{p, \mathrm{PL}}}^{\mathrm{Sync}}(t)<W_{p^{p-s}}^{R}(t)$.

As demonstrated in Fig. 4, in CAD, an adaptive safe extended car-following distance for the PL, $V_{j}^{p, \mathrm{PL}}$, at time $t$ (namely, $d_{V_{j}^{p} \text {,PL }}^{\text {Safe }}(t)$ ) is proposed to keep an absolute safe distance for the platoon. $d_{V_{j}^{p, \text { PL }}}^{\mathrm{Safe}}(t)$ consists of three parts: (i) the moving distance of the response time to brake, denoted by $d_{V_{j}^{p, \mathrm{PL}}}^{\text {Response }}(t)$, (ii) the distance of the bake to a full stop, $d_{V_{j}^{p, \mathrm{PL}}}^{\mathrm{Brake}}(t)$, (iii) the distance required for the transmission of a synchronous message from the preceding platoon that detects a shockwave, $d_{V_{j}^{p, \text { PL }}}^{\text {Sync }}(t)$. That is,

$$
d_{V_{j}^{p, \text { PL }}}^{\mathrm{Safe}}(t) \leftarrow d_{V_{j}^{p, \mathrm{PL}}}^{\text {Response }}(t)+d_{V_{j}^{p, \mathrm{PL}}}^{\mathrm{Brake}}(t)+d_{V_{j}^{p, \mathrm{PL}}}^{\mathrm{Sync}}(t) .
$$

Case 1: Response distance $\left(d_{V_{j}^{p, \mathrm{PL}}}^{\text {Response }}(t)\right)$. A brake may be initialized by a driver or an assistant longitudinal control unit. During the response delay, a moving vehicle is moved forward with the distance of $d_{V_{j}^{\text {p.PL }}}^{\text {Response }}(t)$ before braking,

$$
d_{V_{j}^{p, \mathrm{PL}}}^{\text {Response }}(t)=\beta \cdot \dot{x}_{V_{j}^{p, \mathrm{PL}}}(t),
$$

where $\beta$ represents the response time and $\dot{x}_{V_{j}^{p, \text { PL }}}(t)$ represents the vehicle velocity at time $t$.

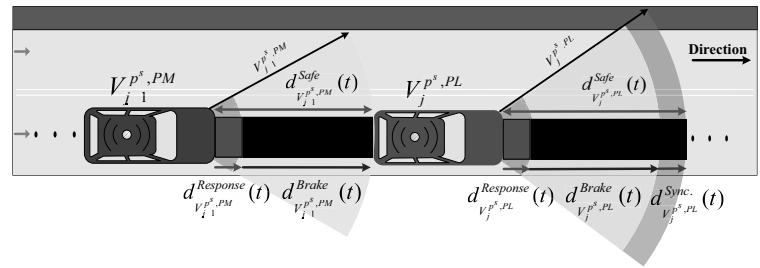

Fig. 4. Safe extended car-following distance of the PL and PM in $\mathrm{CAD}$. 
Case 2: Brake distance $\left(d_{V_{j}^{p, \mathrm{PL}}}^{\mathrm{Break}}(t)\right)$. Based on the Newton laws of motion, the distance required for a moving vehicle to a full stop with a deceleration of $\dot{x}_{V_{j}^{p} \text {,PL }}(t)$ and the minimum distance of the standstill vehicle, $s^{\text {bumper }}$, can be determined by

$$
d_{V_{j}^{p, \mathrm{PL}}}^{\mathrm{Break}}(t)=\frac{\dot{x}_{V_{j}^{p, \mathrm{PL}}(t)^{2}}}{2 \cdot g \cdot \mu(t)}+s^{\text {bumper }},
$$

where $g$ represents the gravitational acceleration, i.e., $9.8 \mathrm{~m} / \mathrm{s}^{2}$, and $\mu(t)$ represents the coefficient of friction between the tire and road surface at time $t$ (MOTC, 2018). Note that $s^{\text {bumper }}$ is defined as $1 / 2$ or one vehicle length. $s^{\text {bumper }}$ is required even though the $\mathrm{PL}$ is in the moving state.

Case 3: Synchronous message distance $\left(d_{V_{j}^{p, \mathrm{PL}}}^{\mathrm{Sync}}(t)\right)$. The objective of the determination of the synchronous message distance, $d_{V_{j}^{p} \text {,PL }}^{\text {Sync }}(t)$, is for the successor platoon to control the platoon velocity synchronously stably. Thus, the shockwave occurring before will not affect the driving of the succeeding platoons. The transmission delay of the emergency message becomes important in CAD.

In CAD, a PL can detect a shockwave by two methods. First, the PL actively detects a shockwave resulting from the sudden braking of the preceding vehicle. Second, a preceding platoon or vehicle detects that there is a shockwave with a backward propagation speed (succeeding propagation), $W_{p^{p-s}}^{V}(t)$, and then the preceding platoon sends an emergency message with the traffic flow and the traffic density of the precedence, $q_{p^{p}}(t)$ and $k_{p^{p}}(t)$, to notify the succeeding platoon leader. Then, by using the traffic analysis in Eqn. (2), we can obtain the shockwave moves from the preceding platoon to the succeeding platoon with the speed of $W_{p^{p-s}}^{V}(t)$. Thus, the affected range of the shockwave at time $t$, denoted by $W_{p^{p-s}}^{R}(t)$, can be determined by the shockwave speed, $W_{p^{p-s}}^{V}(t)$, and the time headway, which is the elapsed time between the time one vehicle finishes passing a fixed observation point and the instant the next vehicle starts to pass that point

$$
\begin{aligned}
W_{p^{p-s}}^{R}(t)= & W_{p^{p-s}}^{V}(t) \\
& \cdot \frac{x_{V_{i}^{p^{p}, \mathrm{PM}}}(t)-x_{V_{j}^{p^{s}, \mathrm{PL}}}(t)-s^{\mathrm{avg} .}}{\dot{x}_{V_{j}^{p^{s}, \mathrm{PL}}}(t)} .
\end{aligned}
$$

In Eqn. (9), $x_{V_{i}^{p^{p}} \text {,PL }}(t)$ and $x_{V_{j}^{p^{s} \text {,PL }}}(t)$ represent the geographical locations of vehicles $V_{i}^{p^{p}, \mathrm{PM}}$ and $V_{j}^{p^{s}, \mathrm{PL}}$ at time $t$, respectively, $\dot{x}_{V_{j}^{p^{s}} \text {,PL }}(t)$ denotes the velocity of vehicle $V_{j}^{p^{s}}$,PL at time $t$, and $s^{\text {avg }}$ denotes the average vehicle length.

After obtaining the shockwave affection range, the time required for the emergency message transmission from the vehicle detecting the shockwave to the succeeding platoon can be computed by the hop-to-hop transmission delay $\left(D_{h 2 h}\right)$,

$$
D_{h 2 h}=\left(D_{\text {access }}+D_{\text {trans. }}\right) \cdot\left\lceil\frac{W_{p^{p-s}}^{R}(t)}{T_{x}^{\text {range }}}\right\rceil,
$$

where $T_{x}^{\text {range }}$ is the wireless transmission range, $\left\lceil W_{p^{p-s}}^{R}(t) / T_{x}^{\text {range }}\right\rceil$ is the number of hops and the transmission time of a single wireless hop at time $t$ consisting of two parts: $\left(D_{\text {access }}+D_{\text {trans. }}\right)$. Note that, in the single hop transmission time, we only consider the access delay $D_{\text {access }}$ and the packet transmission delay $D_{\text {trans }}$, but neglect the queueing delay $D_{\text {queue }}$, the propagation delay $D_{\text {propagation }}$ and the processing delay $D_{\text {processing }}$ because they are too small.

The access delay $D_{\text {access }}$ and the packet transmission delay $D_{\text {trans }}$ are determined as follows. First, for $D_{\text {access, }}$, we assume that CAD VANET adopts IEEE 802.11p with the Enhanced Distributed Channel Access as the wireless interface. EDCA uses CSMA/CA as the channel contention access mechanism. We assume that a packet before successful transmission exhibits an average number of contentions (including the first transmission and the number of re-transmissions), as denoted by $\gamma^{\text {avg }}$. In a contention, the contention delay consists of the AIFS period and the backoff-time. As a result, the access delay $D_{\text {access }}$ can be formulated by (IEEE, 2005)

$$
\begin{aligned}
D_{\text {access }}= & \gamma^{\text {avg. }} \cdot(\text { Backoff Time }+ \text { AIFS }) \\
= & \gamma^{\text {avg. }} \cdot\left[\left(\operatorname{Random}() \cdot \tau_{\text {slot }}\right)\right. \\
& \left.+\left(\operatorname{AIFSN} \cdot \tau_{\text {slot }}+2 \cdot \tau_{\text {slot }}\right)\right] \\
= & \gamma^{\text {avg. }} \cdot\left[\tau_{\text {slot }}(\operatorname{Random}()+\operatorname{AIFSN}+2)\right],
\end{aligned}
$$

where $\operatorname{Random}()$ denotes the randomly waiting delay and $\gamma^{\text {avg }}=p_{c}^{\text {avg }} \gamma^{\max } \cdot p_{c}^{\text {avg }}$ is the average collision probability while $\gamma^{\max }$ is the maximum retry limit. Moreover, AIFSN uses two slots. Note that one slot time is $16 \mu \mathrm{s}$ in IEEE 802.11p (Özturk and Mišić, 2011).

Second, for $D_{\text {trans }}$, it can be determined by $D_{\text {trans. }}=$ $1 / T_{x}^{\text {rate }}$, where $l$ means the packet lengths in bits and $T_{x}^{\text {rate }}$ means the wireless transmission rate.

Finally, the PL of the succeeding platoon moves the distance required for the transmission of a synchronous message from the preceding vehicle or platoon by

$$
d_{V_{j}^{p^{s}, \mathrm{PL}}}^{\mathrm{Sync}}=D_{h 2 h} \cdot \dot{x}_{V_{j}^{p^{s}, \mathrm{PL}}}(t) .
$$

Note that, in the analysis, the average collision probability, $p_{c}^{\text {avg }}$, is randomly determined. That is, the access delay and the transmission delay have been considered, and the connectivity of packet transmission is analysed. Clearly, the hop-to-hop transmission delay is not a fixed robust one. 
In Fig. 5, the idea of APS is to synchronize the velocity change of each PM within platoon $p$. Three key factors: (i) determining the dynamic platoon length according to the hop delay, (ii) computing the next synchronization time, and (iii) synchronizing the platoon velocity, should be solved for achieving APS successfully.

First, the message transmission time is limited according to the driving time for the $\mathrm{PL}\left(V_{j}^{p, \mathrm{PL}}\right)$ with a velocity of $\dot{x}_{V_{j}^{p, \text { PL }}}(t)$ as

$$
\left(D_{\text {access }}+D_{\text {trans }}\right)\left\lceil\frac{L_{p}(t+1)}{T_{x}^{\text {range }}}\right\rceil \leq \frac{d_{V_{j}^{p, \text { PL }}}^{\text {Sync }}(t)}{\dot{x}_{V_{j}^{p, \text { PL }}}(t)},
$$

where $L_{p}(t+1)$ means the platoon length at time $t$ and $\left(D_{\text {access }}+D_{\text {trans }}\right)$ denotes one hop delay. As a result, the platoon length $L_{p}(t+1)$, is described as

$$
L_{p}(t+1) \leq \frac{d_{V_{j}^{p, \text { PL }}}^{\mathrm{Sync}}(t) \cdot T_{x}^{\mathrm{range}}}{\dot{x}_{V_{j}^{p, \text { PL }}}(t) \cdot\left(D_{\text {access }}+D_{\text {trans }}\right)} .
$$

Second, after obtaining the platoon length, the synchronization for the next time for PMs of platoon $p$, denoted by $\tau_{V_{j}^{p, \text { PL }}}^{\text {Sync }}$, can be determined by a PL,

$$
\tau_{V_{j}^{p, \mathrm{PL}}}^{\mathrm{Sync}}=T_{\text {curr }}+\frac{d_{V_{j}^{\text {, } \mathrm{PL}}}^{\mathrm{Sync}}(t)}{\dot{x}_{V_{j}^{p, \mathrm{PL}}(t)}},
$$

where $T_{\text {curr }}$ is the current time when the platoon control message is sent. Note that the synchronization information sent by the platoon leader includes: (i) the platoon ID, (ii) the PL ID and position, (iii) synchronization time $\tau_{V_{j}^{p, \text { PL }}}^{\text {Sync }}$, and (iv) limited speed $\bar{v}_{p}^{\max }\left(\tau_{V_{j}^{p, \text { PL }}}^{\text {Sync }}\right)$. Note that the synchronization length should be shorter than the shockwave affection range.

Consequently, the adaptive platoon velocity for $V_{j}$ at the next synchronization time, $\tau_{V_{j}^{p, \text { PL }}}^{\text {Sync }}, \dot{x}_{V_{j}}\left(\tau_{V_{j}^{p, \text { PL }}}^{\text {Sync }}\right)$, can be obtained,

$$
\dot{x}_{v_{j}}\left(\tau_{V_{j}^{p, \text { pL }}}^{\mathrm{Sync}}\right)=\dot{x}_{v_{J}}(t)+a_{V_{j}} \cdot \Delta t,
$$

where $\Delta t$ represents the time interval of synchronization. The algorithm of Phase 2 (SAD) is demonstrated as Algorithm 2.

\subsection{Phase 3: Adaptive time to collision (ATC) anal-} ysis. After synchronizing platoon driving, the negative impact of shockwave is reduced significantly. Now, in Phase 3, computation of the adaptive time to collision (ATC) is proposed for two adjacent platoons or vehicles. By using the cloud computing/MEC-based

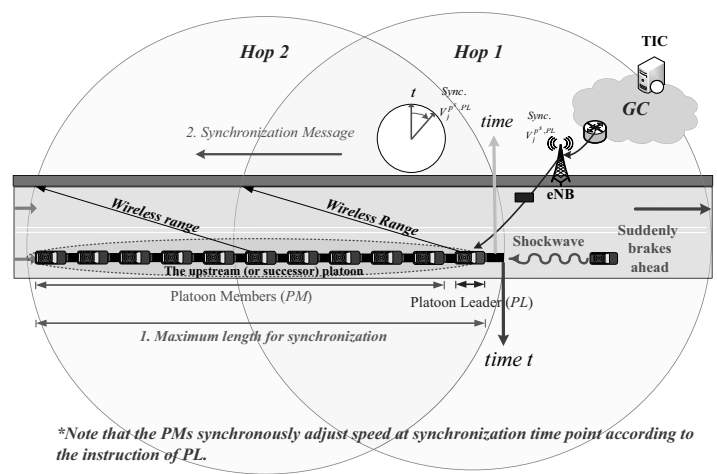

Fig. 5. Adaptive platoon synchronization in CAD.

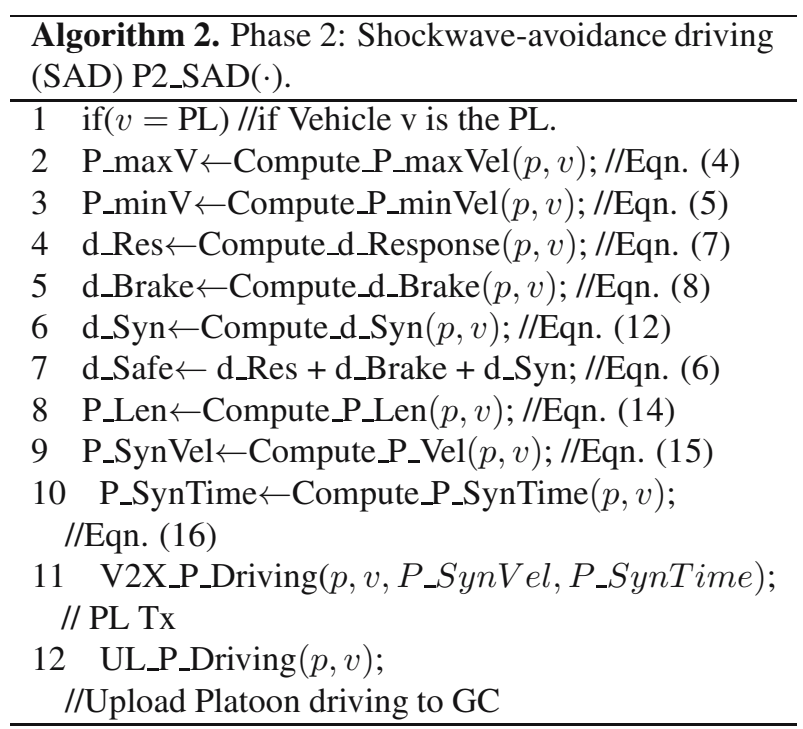

ATC computing mechanism, each platoon and vehicle equipped with the system can accurately receive the TTC, and then the dynamic controls the platoon velocity to achieve safe driving by minimizing driving threats.

In the macroscopic view point, the $\mathrm{road} /$ traffic/platoon information gathered from the vehicular cloud, MEC and the global cloud is adopted as the input parameter for threaten analysis. In Phase 3, the adaptive time to collision of all directly adjacent platoons and vehicles is analyzed according to the sensed/gathered information received from the cloud/MEC.

Assume that the target vehicle $i$ is on $\operatorname{road} r$, and then the average velocity $\left(\bar{x}_{i}^{r}\right)$, location (or point) and travel time $\left(t_{i}^{r}\right)$ of vehicle $i$ can be determined below. The average velocity of vehicle $i$ on road $r$ can be determined by

$$
\overline{\dot{x}_{i}^{r}}=\mu_{r}\left(1-\rho_{r}\right),
$$

where $\mu_{r}$ is the maximum safe speed of road $r$ and $\rho_{r}$ is the traffic density $\left(0 \leq \rho_{r} \leq 1\right)$. A higher traffic density leads to a slower average speed, and vice versa. The travel 


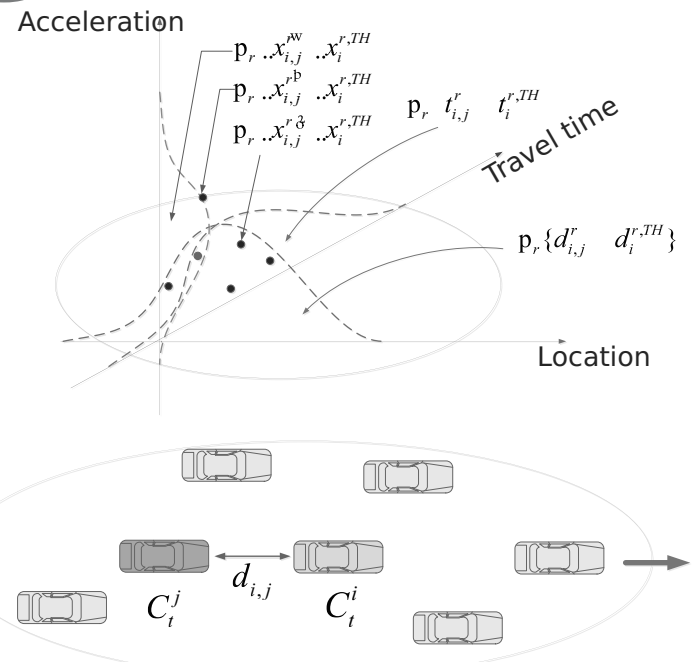

Fig. 6. Threat classifications of the relative parameters of the velocity, location (or point) and travel time.

time of a vehicle on road $r$ can be determined by

$$
t_{i}^{r}=\frac{d_{r}}{{\dot{\dot{x}_{i}^{r}}}_{i}}
$$

where $d_{r}$ is the length of road $r$.

Based on the threat analysis by Brännström et al. (2013), the relative parameters of acceleration, location (or point) and the travel time of the target vehicle $i$ and the adjacent vehicle $j$ can be obtained, as denoted by Relative parameters: $\left\{d_{i, j}^{r}, \ddot{x}_{i, j}^{r}, t_{i, j}^{r}\right\}$, and they can be adopted to classify the threat degree. Then, extended from set invariance theory (Falcone et al., 2011), the thresholds of the relative parameters of target vehicle $i$ can be analyzed and defined as Thresholds: $\left\{d_{i}^{r, \mathrm{TH}}, \ddot{x}_{i}^{r, \mathrm{TH}}, t_{i}^{r, \mathrm{TH}}\right\}$, e.g., $d_{i}^{r, \mathrm{TH}}$ denotes the relative distance threshold of the target vehicle $i$ on road $r$, etc.

Figure 6 shows the threat threshold curves on the relative parameters' axes (i.e., velocity, location and travel time) belonging to the normal distribution. When the parameter value is less than the threshold (i.e., it is inside the curve), the threat exceeds the threshold. For example, in Fig. 3, for the case of the relative distance between vehicles $i$ and $j$ on road $r$ (denoted as $d_{i, j}^{r}$ ), the threat caused by location can be derived based on the normal distribution. Thus, we can determine the distance threshold (denoted by $d_{i}^{r, \mathrm{TH}}$ ), and then define the threat probability caused by location as $p_{r}\left\{d_{i, j}^{r} \leq d_{i}^{r, \mathrm{TH}}\right\}$.

Figure 6 can be adopted to determine the threats caused from the location (or point), the travel time, and the velocity. Based on Bayesian risk theory (Jansson and Gustafsson, 2008), we define the threat index (namely, TI) that consists of these three impact factors of the relative parameters of acceleration, location (or point) and travel time, i.e., $g_{t}^{d_{i, j}^{r}}(t), g_{t}^{\ddot{x}_{i, j}^{r}}(t)$ or $g_{t}^{t_{i, j}^{r}}(t)$, where $g_{t}(t) \in$ $\{0,1\}$. The threat index is defined as

$$
\mathrm{TI}=\frac{g_{t}^{d_{i, j}^{r}}(t)+g_{t}^{\ddot{x}_{i, j}^{r}}(t)+g_{t}^{t_{i, j}^{r}}(t)}{3} .
$$

The impact factors, $g_{t}^{d_{i, j}^{r}}(t), g_{t}^{\ddot{x}_{i, j}^{r}}(t)$, and $g_{t}^{t_{i, j}^{r}}(t)$, are

$$
\begin{aligned}
g_{t}^{d_{i, j}^{r}}(t) & \triangleq\left\{\frac{p_{r}\left\{d_{i, j}^{r} \leq d_{i}^{r, T H} \mid X_{0: n}\right\}}{p_{r}\left\{d_{i, j}^{r}>d_{i}^{r, T H} \mid X_{0: n}\right\}}>c^{\left.d_{i, j}^{r}\right\}},\right. \\
g_{t}^{\ddot{x}_{i, j}^{r}}(t) & \triangleq\left\{\frac{p_{r}\left\{\ddot{x}_{i, j}^{r}>\ddot{x}_{i}^{r, T H} \mid Y_{0: n}\right\}}{p_{r}\left\{\ddot{x}_{i, j}^{r}=\ddot{x}_{i}^{r, T H} \mid Y_{0: n}\right\}}>c^{\left.\ddot{x}_{i, j}^{r}\right\}},\right. \\
g_{t}^{t_{i, j}^{r}}(t) & \triangleq\left\{\frac{p_{r}\left\{t_{i, j}^{r} \leq t_{i}^{r, T H} \mid Z_{0: n}\right\}}{p_{r}\left\{t_{i, j}^{r}>t_{i}^{r, T H} \mid Z_{0: n}\right\}}>c^{t_{i, j}^{r}}\right\} .
\end{aligned}
$$

For instance, in Eqn. (20), $g_{t}^{d_{i, j}^{r}}(t)$ will be set to 1 if the threat probability of $d_{i, j}^{r}$ (i.e., $p_{r}\left\{d_{i, j}^{r} \leq d_{i}^{r, \mathrm{TH}} \mid X_{0: n}\right\}$ ) over the non-threat probability of $d_{i, j}^{r}$ (i.e., $p_{r}\left\{d_{i, j}^{r}>\right.$ $\left.\left.d_{i}^{r, \mathrm{TH}} \mid X_{0: n}\right\}\right)$ is greater than the risk cost of the relative distance, $c^{d_{i, j}^{r}}$. Otherwise, $g_{t}^{d_{i, j}^{r}}(t)$ is set to zero.

In Eqns. (19)-(22), the risk cost of each impact factor (denoted by $c^{d_{i, j}^{r}}, c^{\ddot{x}_{i, j}^{r}}$ or $c^{t_{i, j}^{r}}$ ) is adopted to decide whether the threat caused by the impact factor (i.e., $d_{i, j}^{r}$, $\ddot{x}_{i, j}^{r}$ or $t_{i, j}^{r}$ ) is high or low. The risk cost is defined according to the product of the probability normal density value and non-threat probability. For the case of the relative distance, we define the situation $H_{1}$ is $d_{i, j}^{r} \leq$ $d_{i}^{r, \mathrm{TH}}$ ) (i.e., $H_{1}=d_{i, j}^{r} \leq d_{i}^{r, \mathrm{TH}}$ ) and the situation $H_{2}$ is $d_{i, j}^{r}>d_{i}^{r, \mathrm{TH}}$ ) (i.e., $H_{2}=d_{i, j}^{r}>d_{i}^{r, \mathrm{TH}}$ ), and both occur according to normal distributions. Thus, the risk cost $\left(c^{d_{i, j}^{r}}\right)$ of $d_{i, j}^{r}$ can be defined,

$$
c^{d_{i, j}^{r}}=p\left(H_{1} \mid H_{2}\right) \cdot p\left(H_{2}\right),
$$

where

$$
\begin{aligned}
p & \left(H_{1} \mid H_{2}\right) \\
= & \frac{p\left(H_{1}, H_{2}\right)}{p\left(H_{2}\right)} \\
= & \frac{1}{\sqrt{2 \pi} \sigma_{2} \sqrt{1-\rho^{2}}} \\
& \cdot \exp \left(-\frac{1}{2}\left(\frac{H_{2}-\left[\mu_{2}+\rho \frac{\sigma_{2}}{\sigma_{1}}\left(H_{1}-\mu_{1}\right)\right]^{2}}{\sigma_{2} \sqrt{1-\rho^{2}}}\right)\right) .
\end{aligned}
$$

From the threat classification, one of the important factors is the time to collision (TTC). It specifies that the 


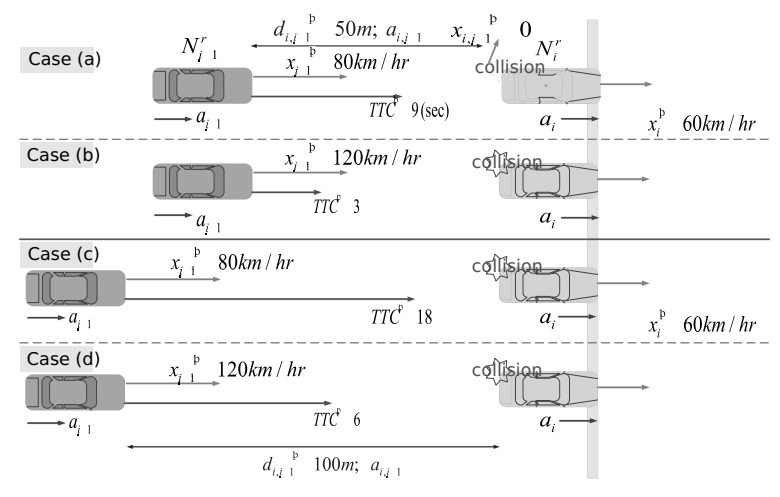

Fig. 7. Examples of determining TTC.

target vehicle will be hit on by the threat vehicle $i+1$ when the TTC expires, if the target vehicle does not employ any threat avoidance mechanism. The TTC can be adopted as the final escape time for the target vehicle $i$. It can be determined by the Newton laws of motion. Before that, we need to determine the relative acceleration $a_{i, i+1}$ by

$$
\begin{gathered}
\left(\dot{x}_{i, i+1}^{c f}\right)^{2}=\dot{x}_{i, i+1}^{\text {init }}(t)^{2}+2 a_{i, i+1} d_{i, i+1}, \\
\because \dot{x}_{i, i+1}^{c f}=0 / / \text { After collision, the final } \\
\quad \text { relative velocity is } 0 . \\
\therefore a_{i, i+1}=\frac{-\dot{x}_{i, i+1}^{\text {init }}(t)^{2}}{2 d_{i, i+1}} .
\end{gathered}
$$

Since these two vehicles move continuously along their moving paths (i.e., their trajectories) on the same lane, the TTC can be determined as

$$
\begin{aligned}
\mathrm{TTC}_{i, i+1} & =\frac{d_{i, i+1}}{\dot{x}_{i, i+1}(t)+\sqrt{\dot{x}_{i, i+1}(t)^{2}+2 d_{i, i+1} a_{i, i+1}}} \\
& =\frac{d_{i, i+1}}{\dot{x}_{i, i+1}(t)},
\end{aligned}
$$

where $\sqrt{\dot{x}_{i, i+1}(t)^{2}+2 d_{i, i+1} a_{i, i+1}}$ is the final velocity.

For example, Fig. 7 demonstrates their TTCs of four different cases. In Cases (a) and (b), the relative distance (i.e., two vehicles or platoons) is $50 \mathrm{~m}$. Since Case (a) deals with a slower relative velocity, Case (a) yields a longer TTC. Cases (c) and (d) have a longer relative distance, TTCs of Cases (c) and (d) are longer than those of Cases (a) and (b). In Case (a), the TTC is determined by $50[\mathrm{~m}] /(20 \cdot 1000 / 3600)=9[\mathrm{sec}]$.

The consecutive steps of Phase 3 (ATC) are presented in Algorithm 3.

\section{Numerical results}

In this section, the proposed CAD approach is evaluated by comparing it with several important techniques:

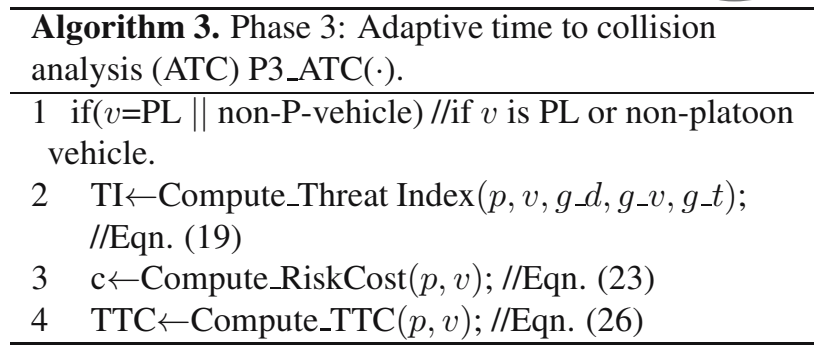

integrated full-range speed assistance (IRSA) (Wilmink et al., 2007), intelligent driver model (IDM) (Treiber et al., 2000), and human driving (HUMAN). IRSA models the driving behavior extended from CACC and determines the driving velocity according to the average velocity of three preceding vehicles. The IDM models the driving behavior extended from ACC and considers the 1.5 second time headway. In the HUMAN driving model, we consider some parameters: (i) delayed response time, (ii) random response time (12 seconds) for determining the car-following distance, etc., to simulate driving behaviors

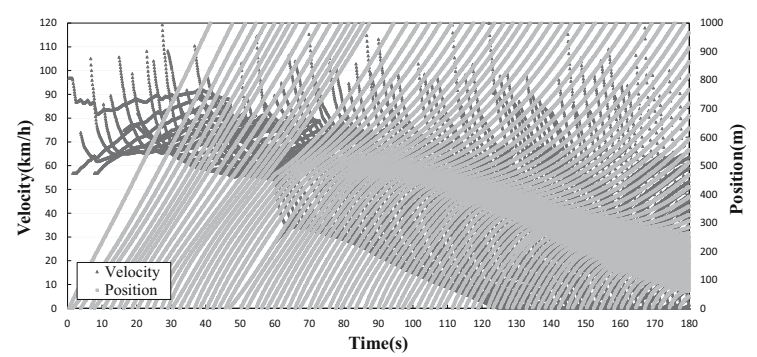

(a) HUMAN

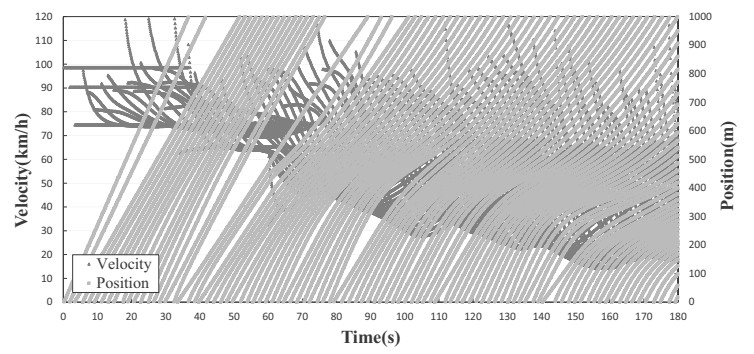

(b) IRSA

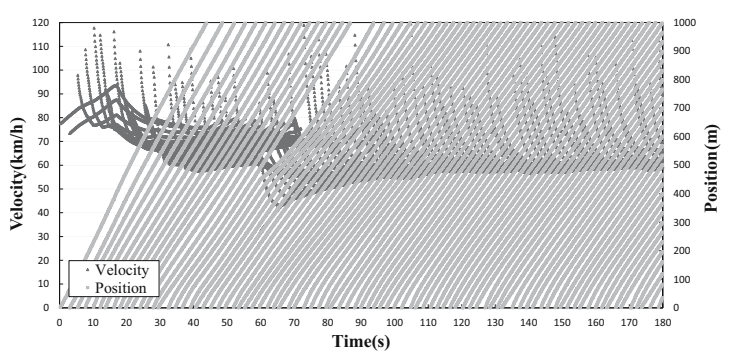

(c) $\mathrm{CAD}$

Fig. 8. Velocity joint position trajectory. 
by (MicroSim, 2018).

The evaluated network model is a two-lane one-kilometer-long road, in which the average vehicle length is set to 6 meters and the minimum distance of the standstill vehicle is set to 3 meters. In the traffic model, the incoming vehicles initiated at the start-end point are generated by the Poisson distribution with arrival rate (Thompson, 2001), and the inter-vehicle distance is formulated by the IDM with the continuous function (Treiber et al., 2000). The vehicle mobility model is generated by the normal distribution with the average velocity and variance (Tacq, 2010), where the average velocity of evaluations is generated between 80 and 110 $\mathrm{km} / \mathrm{h}$.

Figures 8(a)-(c) demonstrate the velocity joint position trajectory results affected by shockwaves among all compared approaches (CAD, ISRA, and HUMAN), in which the sudden braking events happen at the 60th second. In Fig. 8(a), in the case of HUMAN, the suddenly braking results in all vehicles decreasing their velocities during the time interval from 60 to 125 [s]. Moreover, the event leads to the butterfly effect that decreases the velocity of all affected vehicles to $0 \mathrm{~km} / \mathrm{h}$ during the time interval from 125 to 180 [s]. In Fig. 8(b), in the case of IRSA, a vehicle refers to the velocities of (at most) three adjacent leading vehicles. The following vehicles can thus decrease their velocity priorly. The velocities of all vehicles are all above $14 \mathrm{~km} / \mathrm{h}$. However, without cooperation, the velocities of the following vehicles change inconsistently, which results in unstable driving. In Fig. 8(c), CAD adopts the cooperative platoon-based control. The velocities of the following vehicles change consistently, even though it exhibits a suddenly braking at $t=60[\mathrm{~s}]$ and only affects a consistent velocity decreasing during the time interval from 60 to $70[\mathrm{~s}]$. Then, the following vehicles restore the velocities up to $70 \mathrm{~km} / \mathrm{h}$. That is, CAD can effectively avoid the butterfly effects resulting from a suddenly braking.

Figures 9(a)-(b) show the 3D of the velocity joint position trajectory results of ISRA and CAD, in which the sudden braking events happen at every interval of 60 [s]. In Fig. 9(a), ISRA yields obvious velocity changes while exhibiting sudden brakes. IRSA suffers from asynchronous changes of the velocities of neighbor vehicles, and then significantly decreases the velocity while exhibiting shockwaves. In Fig. 9(b), CAD outperforms ISRA in the avoidance of butterflies, so the $3 \mathrm{D}$ trajectory of CAD is changed smoothly. The reason is the platoon-based CAD adaptively determines the platoon velocity and the safe distance for platoon synchronization.

Figure 10 compares the number of shockwaves under different traffic loads ranging from 1 to 5 Erlangs. CAD yields no shockwaves, but HUMAN yields the highest number of shockwaves. Furthermore, IRSA yields a fewer number of shockwaves than IDM. Moreover, the number

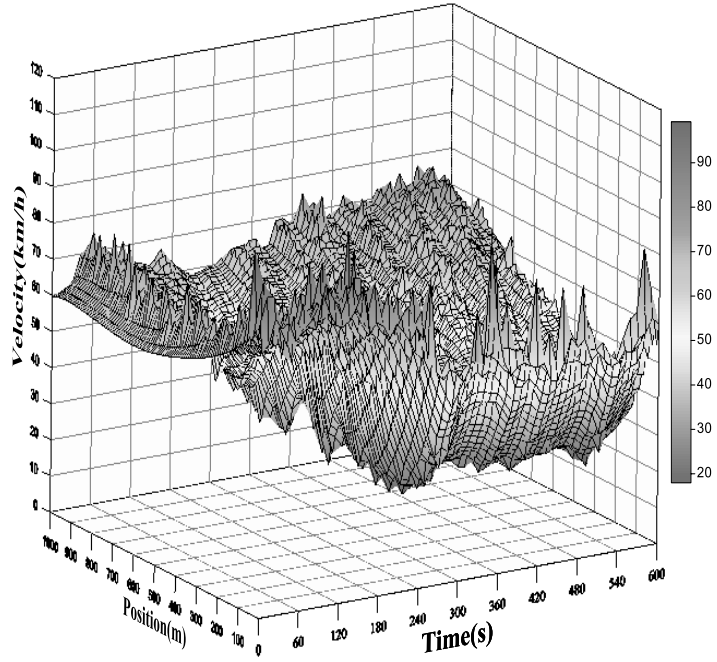

(a) IRSA

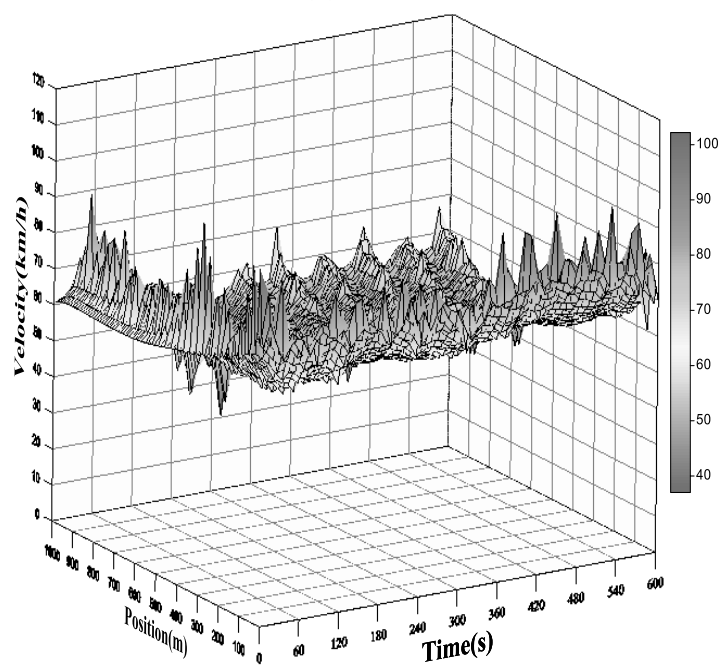

(b) $\mathrm{CAD}$

Fig. 9. 3D velocity joint position trajectory.

of shockwaves of all compared approaches (except CAD) increases as the traffic load increases.

Figure 11 compares the average vehicle velocities of all compared approaches under different traffic loads ranging from 1 to 5 Erlangs. The velocities of all approaches decrease as the traffic load increases. CAD yields a higher velocity, but HUAMN and IDM yield a lower one. The reason is that CAD exhibits a stable velocity by using cooperative platoon-based driving, so the vehicles synchronously change their velocities. However, HUMAN and IDM lack the cooperative CACC mechanism; the vehicle velocity is changed inconsistently and results in unstable velocity and a lower average velocity.

Figure 12 demonstrates the TTCs of simulation and analysis of the proposed CAD approach under a vehicle 


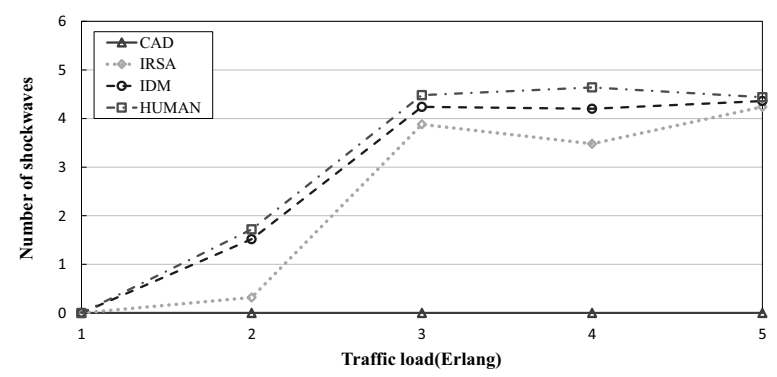

Fig. 10. Number of shockwaves under different traffic loads.

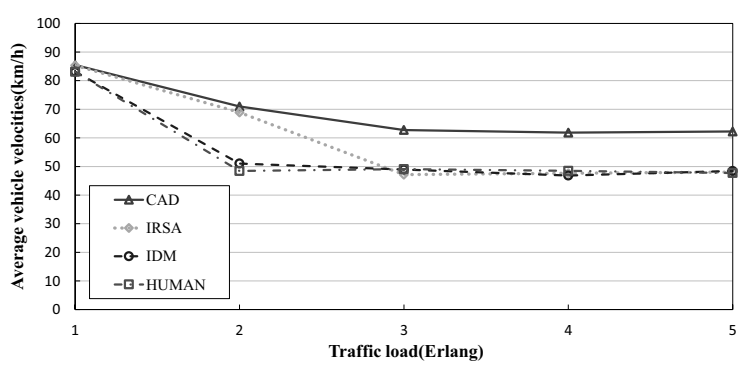

Fig. 11. Average vehicle velocities under different traffic loads.

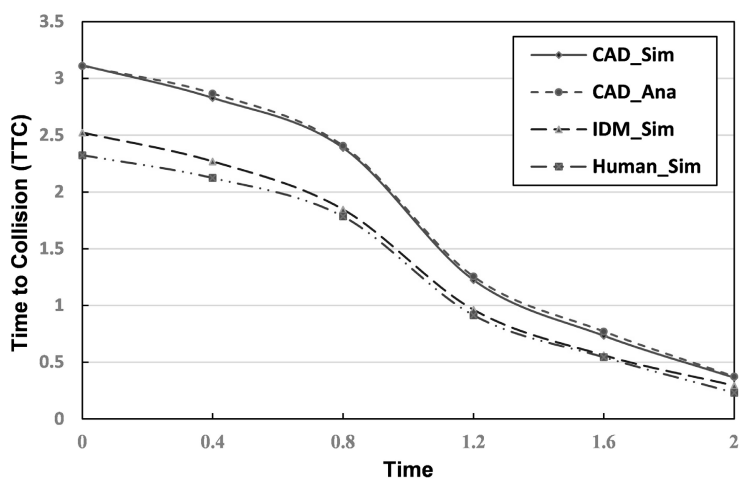

Fig. 12. TTCs of simulation and analysis [s].

velocity of $60 \mathrm{~km} / \mathrm{h}$. The TTCs of simulation and analysis are very close, which can justify the correctness of the analytical model. Moreover, the TTCs of both simulation and analysis decrease as the evaluation time increases. The reason is the number of vehicles on roads increases, and then decreases the inter-vehicle (or platoon) distance and reduces TTC.

\section{Conclusions and future works}

The intelligent transportation system aims to achieve actively safe driving, lower travel times, stable vehicle velocities, etc. Without using cooperative-based active safe driving, the above-mentioned objectives are difficult to fulfill. However, the cooperative active safe driving mechanism suffers from sudden braking, which easily leads to butterfly effects and brings several challenges: shockwaves, unstable driving, unsafe driving, long travel times, etc. Therefore, this paper proposed the mobile edge computing-based CAD approach to minimize butterfly effects and shockwaves, and then achieve active safe driving.

The main contributions of CAD include (i) adaptive determining of the platoon velocity according to the traffic information, (ii) determining the safe distance for platoon synchronization, (iii) adaptive determining of the platoon length, (iv) avoiding butterfly effects and shockwaves, etc. Numerical results demonstrate that the CAD outperforms the compared approaches in the number of shockwaves and average velocity. In addition, CAD is able to determine the adaptive platoon length according to traffic information gathered from the global and local clouds. Many aspects of analyzing cooperative adaptive platoon driving require a further study. For instance, we are currently extending our model to analyze butterfly effects and shockwaves of dynamic traffic involved in ITS networks cooperating with 5G, MEC, and Cloud.

\section{Acknowledgment}

This research was supported in parts by the Ministry of Science and Technology of Taiwan, ROC, under the grants

- MOST-106-2221-E-194-021-MY3,

- MOST-107-2221-E-224-009-MY2,

- MOST-105-2221-E-224-031-MY2,

- MOST-106-2511-S-252-001, and

- MOST-106-3011-F-252-001.

\section{References}

3GPP (2019). Study on NR Vehicle-to-Everything (V2X), 3GPP Specification 38.885 .

Abuelela, M. and Olariu, S. (2010). Taking VANET to the clouds, Proceedings of the International Conference on Advances in Mobile Computing and Multimedia, Paris, France, pp. 6-13.

Asadi, B. and Vahidi, A. (2011). Predictive cruise control: Utilizing upcoming traffic signal information for improving fuel economy and reducing trip time, IEEE Transactions on Control Systems Technology 19(3): 707-714.

Brännström, M., Sandblom, F. and Hammarstrand, L. (2013). A probabilistic framework for decision-making in collision avoidance systems, IEEE Transactions on Intelligent Transportation Systems 14(2): 637-648.

Burke, M. and Williams, J. (2012). Cooperative ITS regulatory policy issues, Discussion paper, Australia National Transport Commission, pp. 1-77. 
Chang, B.-J., Tsai, Y.-L. and Liang, Y.-H. (2017). Platoon-based cooperative adaptive cruise control for achieving active safe driving through mobile vehicular cloud computing, Wireless Personal Communications 97(4): 5455-5481.

Chen, S., Hu, J., Shi, Y. and Zhao, L. (2016). LTE-V: A TD-LTE-based $\mathrm{v} 2 \mathrm{x}$ solution for future vehicular network, IEEE Internet of Things Journal 3(6): 997-1005.

Desjardinsand, C. and Chaib-draa, B. (2011). Cooperative adaptive cruise control: A reinforcement learning approach, IEEE Transactions on Intelligent Transportation Systems 12(4): 1248-1260.

Dinh, T.Q., Tang, J., La, Q.D. and Quek, T.Q.S. (2017). Offloading in mobile edge computing: Task allocation and computational frequency scaling, IEEE Transactions on Communications 65(8): 3571-3584.

Falcone, P., Ali, M. and Sjöberg, J. (2011). Predictive threat assessment via reachability analysis and set invariance theory, IEEE Transactions on Intelligent Transportation Systems 12(4): 1352-1361.

Geroliminis, N. and Skabardonis, A. (2011). Identification and analysis of queue spillovers in city street networks, IEEE Transactions on Intelligent Transportation Systems 12(3): 1107-1115.

Gomes, A.S., Sousa, B., Palm, D.V., Fonseca, A., Zhao, Z., Monteiro, E., Braun, T., Simoes, P. and Cordeiro, L. (2017). Edge caching with mobility prediction in virtualized LTE mobile networks, Future Generation Computer Systems 70(1): 148-162.

Helbing, D. (2001). Traffic and related self-driven many-particle systems, Reviews of Modern Physics 73(4): 1067-1141.

Huang, C.-L., Fallah, Y., Sengupta, R. and Krishnan, H. (2010). Adaptive intervehicle communication control for cooperative safety systems, IEEE Network 24(1): 6-13.

Hwang, R.-H., Chang, B.-J., Tsai, Y.-L. and Liang, Y.-H. (2017). Mobile edge computing-based vehicular cloud of cooperative adaptive driving for platooning autonomous self driving, Proceedings of IEEE SC2 2017, Kanazawa, Japan, pp. 33-39.

IEEE (2005). Part 11: Wireless LAN medium access control (MAC) and physical layer (PHY) specifications amendment: Medium access control (MAC) quality of service enhancements, Technical report, IEEE Std. 802.11e, IEEE, Piscataway, NJ.

IEEE (2011). IEEE standard for information technology-telecommunication and information exchange between systems-local and metropolitan area networks-specific requirements, Technical report, IEEE Std. 802.11p, IEEE, Piscataway, NJ.

IEEE (2013). IEEE trial-use standard for wireless access in vehicular environments (wave)-multi-channel operation, Technical report, IEEE Std. P1609.4, IEEE, Piscataway, NJ.

ITS (2018). Intelligent transportation systems (ITS), US Department of Transportation, Washington, DC, http:/ /www.its.dot.gov/
Jansson, J. and Gustafsson, F. (2008). A framework and automotive application of collision avoidance decision making, Automatica 44(9): 2347-2351.

Kuhne, R. and Michalopoulos, P. (1997). Continuum flow models, in N.H. Gartner et al. (Eds.), Traffic Flow Theory: A State of the Art Report-Revised Monograph on Traffic Flow Theory, Oak Ridge National Laboratory, Oak Ridge, TN.

Lorenz, E. (1963). Deterministic nonperiodic flow, Journal of the Atmospheric Sciences 20(2): 130-141.

Maag, C., Mühlbacher, D., Mark, C. and Krüger, H.-P. (2012). Studying effects of advanced driver assistance systems (ADAS) on individual and group level using multi-driver simulation, IEEE Intelligent Transportation Systems Magazine 4(3): 45-54.

MicroSim (2018). Microsimulation of road traffic flow, http: //www.traffic-simulation.de/

Milanés, V., Alonso, J., Bouraouiand, L. and Ploeg, J. (2011a). Cooperative maneuvering in close environments among cybercars and dual-mode cars, IEEE Transactions on Intelligent Transportation Systems 12(1): 15-24.

Milanés, V., Godoy, J., Villagra, J. and Perez, J. (2011b). Automated on-ramp merging system for congested traffic situations, IEEE Transactions on Intelligent Transportation Systems 12(2): 500-508.

Mitropoulos, G.K., Karanasiou, I.S., Hinsberger, A., Aguado-Agelet, F., Wieker, H., Hilt, H.-J., Mammarand, S. and Noecker, G. (2010). Wireless local danger warning: Cooperative foresighted driving using intervehicle communication, IEEE Transactions on Intelligent Transportation Systems 11(3): 539-553.

MOTC (2018). Ministry of Transportation and Communications (MOTC), Taipei, http: / /www. iot.gov.tw/.

Mousannif, H., Khalil, I. and Moatassime, H.A. (2011). Cooperation as a service in VANETs, Journal of Universal Computer Science 17(8): 1202-1208.

Özturk, S. and Mišić, J. (2011). On non-saturation regime in IEEE $802.11 \mathrm{p}$ based VANET with mobile nodes, Proceedings of IEEE PIMRC, Toronto, Canada, pp. 740-744.

Papadimitratos, P., La Fortelle, A., Evenssen, K., Brignolo, R. and Cosenza, S. (2009). Vehicular communication systems: Enabling technologies, applications, and future outlook on intelligent transportation, IEEE Communications Magazine 47(11): 84-95.

Pauwelussen, J. and Feenstra, P.J. (2010). Driver behavior analysis during ACC activation and deactivation in a real traffic environment, IEEE Transactions on Intelligent Transportation Systems 11(2): 329-338.

Satria, D., Park, D. and Jo, M. (2017). Recovery for overloaded mobile edge computing, Future Generation Computer Systems 70(1): 138-147.

Schakel, W., Arem, B. and Netten, B. (2010). Effects of cooperative adaptive cruise control on traffic flow stability, Proceedings of IEEE Conference on Intelligent Transportation Systems, ITSC 2016, Rio de Janeiro, Brazil, pp. 759-764. 
Somda, F. and Cormerais, H. (2011). Auto-adaptive and string stable strategy for intelligent cruise control, IET Intelligent Transport Systems 5(3): 168-174.

Stevenson, R. (2011). A drivers sixth sense, IEEE Spectrum 48(10): 50-55.

Tacq, J. (2010). The normal distribution and its applications, in E. Baker et al. (Eds.), International Encyclopedia of Education, 3rd Edition, Elsevier Science, Amsterdam.

Taleb, T., Benslimane, A. and Letaief, K. (2010). Toward an effective risk-conscious and collaborative vehicular collision avoidance system, IEEE Transactions on Vehicular Technology 59(3): 1474-1486.

Thompson, W.J. (2001). Poisson distributions, Computing in Science and Engineering 3(3): 78-82.

Treiber, M., Hennecke, A. and Helbing, D. (2000). Congested traffic states in empirical observations and microscopic simulations, Physical Review E62 10(1103): 1805-1824.

Wilmink, I.R., Klunder, G.A. and Arem, B. (2007). Traffic flow effects of integrated full-range velocity assistance (IRSA), Proceedings of IEEE Intelligent Vehicles Symposium, IVS 2007, Istanbul, Turkey, pp. 1204-1210.

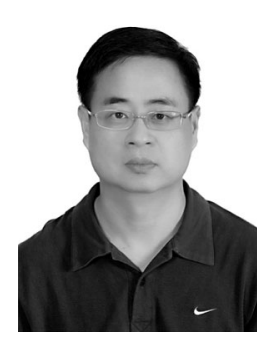

Ben-Jye Chang received the $\mathrm{PhD}$ degree in computer science and information engineering from National Chung-Cheng University, Taiwan, in 2001 and the MSc degree in computer engineering from the University of Massachusetts, Lowell, USA, in 1992. He is currently a distinguished professor of the Department of Computer Science and Information Engineering (CSIE), National Yunlin University of Science and Technology, Yunlin, Taiwan. He was the chair of the CSIE Department in 2011-2014. In 2008 he was also the chair of the CSIE Department of the Chaoyang University of Technology, Taichung, Taiwan, which he joined in 2002. His research interests include 5G cooperative communication, 4G LTE/LTE-A, optimal cooperative communication, relaying and small cell technologies, cloud computing, SDN/NFV-based flow networking, mobile edge computing, 5G WiFi, cloud active safe driving, IoT/IoV, cross layer-based mechanism, wireless real-time transmission congestion control, and QoS-based wireless relay-based cooperative networking.

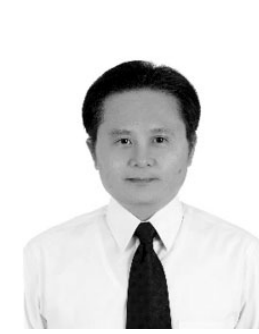

Ren-Hung Hwang received his $\mathrm{PhD}$ degree in computer science from the University of Massachusetts, Amherst, USA, in 1993. Then he joined the Department of Computer Science and Information Engineering, National Chung Cheng University, Chia-Yi, Taiwan, where he is now a distinguished professor. He served as the dean of the College of Engineering in 2014-2017. He is currently on the editorial boards of the Journal of Information Science and Engineering and the International Journal of Ad Hoc and Ubiquitous Computing. Prof. Hwang has published more than 200 international journal and conference papers. He is also a co-author of the textbook Computer Networks: An Open Source Approach (www . mhhe . com/ I in), with Ying-Dar Lin and Fred Baker (McGraw-Hill, 2011). He received the IEEE Best Paper Award at IEEE SC2 2017 and IEEE IUCC 2014, and the IEEE Outstanding Paper Award at IEEE IC/ATC/ICA3PP 2012. His current research interest is in the Internet of things, cloud computing, and software defined networks.

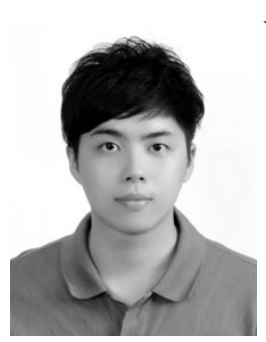

Yueh-Lin Tsai received his MSc degree in computer science and information engineering from the National Yunlin University of Science and Technology, Taiwan, in 2013. His current research interests include platoon-based active safe driving analysis, intelligent transportation mechanisms, radio resource management of cellular communications, and 4G LTE-advanced technology.

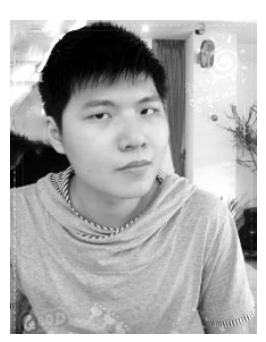

Bo-Han Yu received his MSc degree in information and communication engineering from the National Yunlin University of Science and Technology, Taiwan, in 2014. His current research interests include wireless networking, performance analysis and 4G LTE-advanced technology.

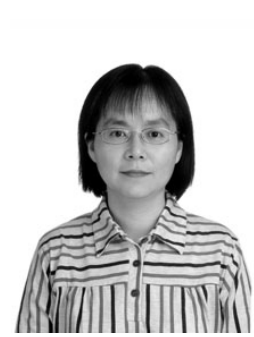

Ying-Hsin Liang received the MSc degree in electrical engineering from the University of Massachusetts, Lowell, USA, in 1993 and the $\mathrm{PhD}$ degree in industrial education and technology from the National Changhua University of Education, Changhua, Taiwan, in 2005. She is currently a full professor with the Department of Multimedia Animation and Application, Nan Kai University of Technology, Taiwan, which she joined in 1993. Her research interests include wireless networking, mobile communications, technological and engineering education, web-based and multimedia learning, and gerontechnology.

Received: 31 July 2018

Revised: 1 January 2019

Accepted: 1 February 2019 